\title{
COVID-19 revisiting inflammatory pathways of arthritis
}

\section{Georg Schett $₫$, Bernhard Manger, David Simon $(\mathbb{D}$ and Roberto Caporali}

Abstract | Coronavirus disease 2019 (COVID-19) is an infectious disease, caused by severe acute respiratory syndrome coronavirus 2 , which predominantly affects the lungs and, under certain circumstances, leads to an excessive or uncontrolled immune activation and cytokine response in alveolar structures. The pattern of pro-inflammatory cytokines induced in COVID-19 has similarities to those targeted in the treatment of rheumatoid arthritis. Several clinical studies are underway that test the effects of inhibiting IL-6, IL-1 $\beta$ or TNF or targeting cytokine signalling via Janus kinase inhibition in the treatment of COVID-19. Despite these similarities, COVID-19 and other zoonotic coronavirus-mediated diseases do not induce clinical arthritis, suggesting that a local inflammatory niche develops in alveolar structures and drives the disease process. COVID-19 constitutes a challenge for patients with inflammatory arthritis for several reasons, in particular, the safety of immune interventions during the pandemic. Preliminary data, however, do not suggest that patients with inflammatory arthritis are at increased risk of COVID-19.

Severe acute respiratory syndrome coronavirus 2 (SARS-CoV-2) is the third zoonotic coronavirus to cross the species barrier, infect humans and become transmitted between humans ${ }^{1}$. Whereas the two other zoonotic coronaviruses, SARS-CoV and Middle East respiratory syndrome coronavirus (MERS-CoV), could be contained at the regional level, SARS-CoV-2 has led to a global pandemic known as coronavirus disease 2019 (COVID-19). Although COVID-19 usually presents as a mild respiratory disease, like infections caused by the four endemic human coronavirus (HCoV-229E, HCoV-NL63, HCoV-OC43 and $\mathrm{HCoV}-\mathrm{HKU} 1$ ), it can occasionally lead to severe alveolar inflammation. In a large study from China, $\sim 15 \%$ of SARS-CoV-2-infected patients developed shortness of breath, radiological infiltrates in the lung and a drop in blood oxygen saturation $(<93 \%)$, and $5 \%$ had critical illness requiring ventilation ${ }^{2}$. Although such data might slightly overestimate the frequency of severe lung involvement, because testing for SARS-CoV-2 infection is not performed in milder cases and is therefore underestimated, COVID-19 undoubtedly constitutes a substantial risk factor for pulmonary failure. Histopathological findings of the lungs of deceased patients with COVID-19 showed extensive alveolar damage, fibrin deposits, widespread infiltration with immune cells and thrombosis of small and large pulmonary vessels ${ }^{3}$. Even though the clinical picture of severe COVID-19 resembles that of acute respiratory distress syndrome (ARDS), these lung histopathological findings suggest that COVID-19 creates a specific form of alveolar disease that is different from other forms of ARDS. The risk of severe lung disease in the context of COVID-19 depends on certain, as yet to be determined, susceptibility factors of the host. Higher age is one of the known risk factors and, as with the other zoonotic coronaviruses, the clinical course of COVID-19 is more severe in older individuals, whereas children and adolescents, who typically develop flu-like symptoms owing to the four endemic coronaviruses, are virtually spared from SARS and MERS ${ }^{4}$. Reports suggest, however, that in very rare cases, Kawasaki-like disease can occur as a complication of COVID-19 (REF. ${ }^{5}$ ).
The current COVID-19 pandemic raises several interesting research questions, as well as clinical challenges in the context of rheumatic diseases. In this Perspective article, we address some of these points. We first consider the musculoskeletal symptoms elicited by COVID-19. We then describe how the mechanisms of alveolar inflammation in COVID-19 share similarities with those in rheumatoid arthritis (RA), in particular, with respect to the pattern of cytokines involved in driving the inflammatory process. On the basis of this concept, we discuss the potential of currently existing cytokine-blocking strategies to treat COVID-19, as well as their respective impact on the risk of developing viral infection. Finally, we critically discuss the effect of the COVID-19 pandemic on the treatment of patients with rheumatic diseases.

\section{Coronavirus and rheumatic symptoms}

Musculoskeletal symptoms can develop during coronavirus infections, as with other respiratory infections. In general, viral infections can cause arthritis, but the spectrum of symptoms is wide, ranging from arthralgia to spurious and chronic arthritis $^{6}$ (FIG. 1). Whereas chronic arthritis can be caused by hepatitis $C$ and several endemic alphaviruses (such as Chikungunya, Ross River, Barmah Forest, Sindbis, O'nyongnyong and Mayaro viruses), self-limited arthritis occurs with parvovirus B19, rubella or hepatitis B virus 6 . By contrast, coronaviruses do not typically cause clinical arthritis but rather arthralgia and myalgia ${ }^{7-11}$. Joint and muscle pain are quite rare (occurring in $<10 \%$ of cases) in the endemic coronaviruses, whereas arthralgia is reported in 15\% of patients with COVID-19, and myalgia is even more frequent $(44 \%)^{7,8}$. Musculoskeletal symptoms, however, do not seem to be associated with COVID-19 severity ${ }^{8}$. Myalgia is also common in SARS-CoV and is found in $49-68 \%$ of cases $^{9,10}$, but arthralgia is less frequent (11\%). Arthralgia and myalgia are also common in patients with MERS-CoV $(32 \%)^{11}$. However, it needs to be considered that these prevalence data are based on the background of high doses of glucocorticoids (cumulative doses of $>1 \mathrm{~g}$ ). Glucocorticoids do not seem to be of benefit 


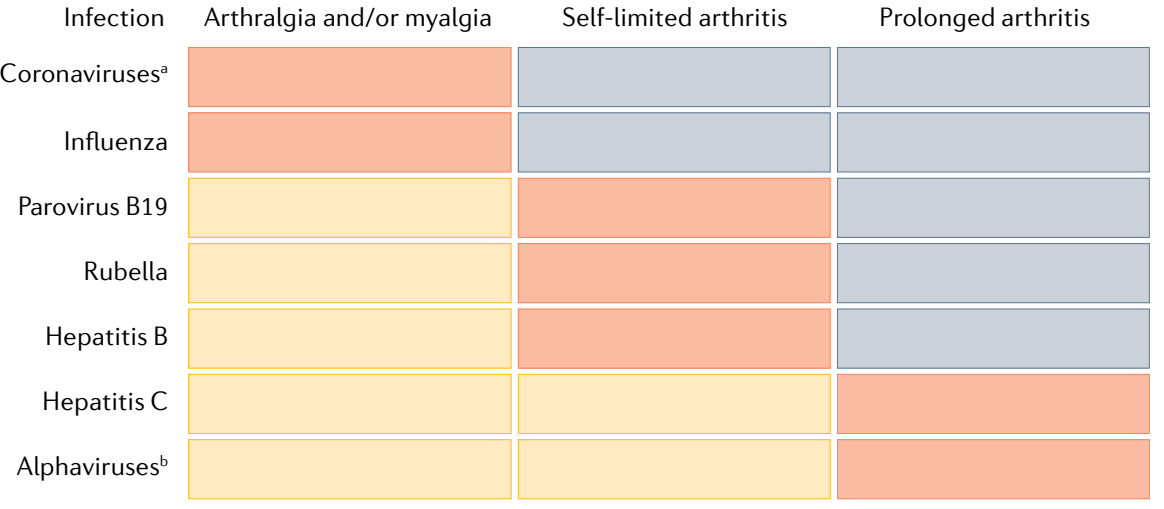

Frequently reported

Sometimes reported

$\square$ Not reported

Fig. 1 | Range of arthritis symptoms associated with infections. Musculoskeletal symptoms that can develop during viral infections, including coronavirus infections, range from arthralgia to chronic arthritis. ${ }^{a}$ Coronaviruses refer to the zoonotic coronaviruses severe acute respiratory syndrome coronavirus (SARS-CoV), SARS-CoV-2 and Middle East respiratory syndrome CoV (MERS-CoV).

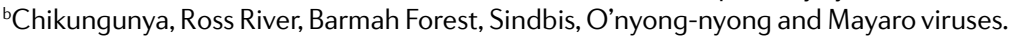

in SARS or MERS ${ }^{12-14}$, but they probably dampen the musculoskeletal manifestations of COVID-19. Overall, COVID-19 seems to present with or be complicated by mild-to-modest musculoskeletal symptoms, which are indistinguishable from those associated with other respiratory viruses, such as influenza infection. Finally, another interesting aspect in the relationship between coronaviruses and arthritis is that the endemic human coronaviruses are associated with an increased risk of developing RA. In a large Korean study, the authors observed that infections with endemic human coronavirus, parainfluenza virus and metapneumovirus coincided with an increased rate of development of $\mathrm{RA}^{15}$. Thus, the COVID-19 pandemic could potentially lead to an increase in cases of RA. However, to date, there is no report to indicate that people develop autoimmune inflammatory arthritis, such as RA, after being infected by SARS-CoV-2 or any of the other six known human coronaviruses.

\section{Alveolar inflammation in COVID-19}

Although some data suggest that immune cells such as macrophages can be infected by SARS viruses, the primary target of these viruses is epithelial cells. Infection is enabled by cellular expression of angiotensinconverting enzyme 2 (ACE2), a carboxypeptidase that binds to the spike proteins of SARS-CoV and SARS-CoV-2; ACE2 is expressed by the epithelium of the mouth, tongue and upper airways, thus enabling endocytosis of the virus ${ }^{16}$. In most patients, unknown host factors confine the infection to these structures, leading to flu-like symptoms. ACE2 can be shed from epithelial cells by the activity of the metalloproteinase ADAM17 (REF. ${ }^{17}$ ), which enables ACE2 to act as a decoy receptor for SARS-CoV-2, potentially preventing viral spreading. The most critical step in SARS and MERS invasion is the infection of alveolar type 2 (AT2) cells, which also express ACE2. This process can lead to the acute alveolar damage common to all zoonotic coronaviruses ${ }^{18}$. AT2 cells are important for alveolar regeneration after damage, in which setting their proliferation depends on IL- $1 \beta$ and TNF expressed by resident macrophages and other immune cells ${ }^{19}$.

In some patients with SARS or MERS, this inflammatory alveolar niche gets out of control (FIG. 2). Age is by far the most relevant risk factor for severe COVID-19. It is well known that cytokine responses, such as IL-6 responses, are increased in advanced age ${ }^{20}$. At the alveolar level, transcriptional profiling of AT2 cells from the lungs of aged mice has shown that these cells have increased transcriptional instability, which leads to disrupted cell function and more widespread responses ${ }^{21}$. This process might enable the triggering of excessive or uncontrolled cytokine responses, also referred to as a 'cytokine storm', in the lungs of patients infected with SARS or MERS. In support of this concept, circulating serum concentrations of most pro-inflammatory effector cytokines, such as TNF, IL-1 $\beta$, IL-6, IL-8, granulocyte colony-stimulating factor (G-CSF) and granulocyte-macrophage colony-stimulating factor (GM-CSF), as well as chemokines, such as CCL2 (also known as MCP1), CXCL10 (also known as IP-10) and CCL3 (also known as MIP1a), are elevated in patients with COVID-19, resembling over-activation of innate immunity ${ }^{22}$. This cytokine activation profile illustrates the accumulation of an armada of neutrophils and bone-marrow-derived macrophages in the damaged alveolar structures.

The hierarchical order of cytokines involved in COVID-19 is not yet known, but mediators that are currently therapeutically targeted in RA and in autoinflammatory syndromes seem to be of central importance ${ }^{23}$. Initially, epithelial cells infected by SARS viruses act as a cytokine source, releasing IL- 6 and IL- 1 as well as IL-8 and several other chemokines that can activate macrophages, attract neutrophils and orchestrate homing of monocytes and macrophages to inflammatory sites ${ }^{24}$. For instance, the nucleocapsid protein of SARS viruses can activate IL-6 expression through the transcription factor NF- $\kappa B^{25}$. After mounting the initial inflammatory response to alveolar damage, monocytes and macrophages seem to contribute to cytokine production. Hence, SARS-CoV stimulates macrophages to increase their production of certain chemokines (for example, MIP1 $\alpha$ ), as well as IL-6 and TNF ${ }^{26}$. This effect further augments the infiltration and activation of neutrophils and macrophages in the alveoli, further contributing to tissue destruction.

Infection with SARS viruses is associated with peripheral lymphocytopenia, which in the case of COVID-19 is present in $>80 \%$ of patients at the time of hospital admission and affects predominantly $\mathrm{CD}^{+} \mathrm{T}$ cells, including effector memory $\mathrm{T}$ cells and regulatory $\mathrm{T}$ cells ${ }^{22}$. The pathogenesis of COVID-19-associated lymphocytopenia is unknown, but it is thought that lymphocytes redistribute to the lungs. Lessons from previous outbreaks of SARS-CoV and MERS-CoV infections in fact suggest that these viruses induce robust IL-17 production by lymphocytes and that IL-17 boosts the pro-inflammatory nature of the host anti-viral immune response to SARS viruses ${ }^{27,28}$. In accordance with this mechanism, concentrations of not only IL-17 but also GM-CSF (another important pro-inflammatory mediator expressed by $\mathrm{T}$ helper $17\left(\mathrm{~T}_{\mathrm{H}} 17\right)$ cells) are high in patients with COVID-19 (REFS ${ }^{22,23}$ ). As IL-17 is involved in the recruitment of neutrophils to inflammatory sites, it is likely that this cytokine - which, alongside TNF, is an important therapeutic target in spondyloarthritis and psoriatic arthritis - might contribute to the development of COVID-19. Notably, IL-17 is not the only $\mathrm{T}$ cell-derived effector cytokine that is elevated in COVID-19 and might contribute 
to lung inflammation. Concentrations of IL-4, IL-9 and IL-13, which are associated with type 2 immunity, bronchial hypersensitivity and increased mucus production, are also high in patients with COVID-19 (REF. ${ }^{22}$ ). These mediators reflect activation of $\mathrm{T}_{\mathrm{H}} 2$ cells, $\mathrm{T}_{\mathrm{H}} 9$ cells, eosinophils and type 2 innate lymphoid cells in the lungs of patients with COVID-19. Although these mediators might have a pro-inflammatory function in the lungs in COVID-19, several previous studies have shown that they act differently in the joints and contribute to resolution of arthritis ${ }^{29,30}$.

\section{Arthritis drugs for COVID-19?}

\section{SARS-CoV-2, like SARS-CoV and}

MERS-CoV, has the capability to trigger a massive immune response associated with a cytokine storm, leading to ARDS, which can be lethal in the most serious cases of COVID-19 (REFS ${ }^{23,31,32}$ ). Hence, anti-cytokine intervention, which does not increase the risk of viral infection and thus might not affect viral clearance but would inhibit the hyper-inflammatory state in COVID-19, might be beneficial ${ }^{33}$. By contrast, broad immunosuppression, especially by targeting lymphocyte function, for example, with glucocorticoid therapy, could be harmful in COVID-19, which itself is associated with substantial lymphocytopenia. Indeed, none of the current data on SARS-CoV-2, SARS-CoV and MERS-CoV supports the use of glucocorticoids ${ }^{12-14}$. Thus, the use of anti-cytokine agents in the most critical phases of COVID-19 is supported by a rationale that overcomes potential concerns about dampening the host response to the virus. Which pro-inflammatory cytokine is most critical to the pathogenesis of COVID-19 is currently unknown, although IL-6 seems to be pivotal ${ }^{34,35}$. Indeed, the results of a Chinese study ${ }^{36}$ exploring the use of the IL-6 receptor (IL-6R) antagonist tocilizumab are encouraging, and several trials with IL-6R antagonists (tocilizumab and sarilumab) and IL-6 inhibitors (sirukumab) have now been initiated ${ }^{37,38}$. IL-1 inhibition also seems to make sense in the treatment of COVID-19, as IL- $1 \beta$ is produced by AT2 cells upon infection with SARS-CoV ${ }^{24}$ and is an important effector cytokine of innate immunity, controlling neutrophil and macrophage function. Indeed, IL- $1 \beta$ inhibition with high-dose anakinra or with canakinumab is currently being evaluated as treatment for COVID-19 $\left(\right.$ REFS $\left.^{39,40}\right)$. TNF has been proposed as a target for the treatment of SARS-CoV ${ }^{41}$ and more recently for SARS-CoV-2 infection ${ }^{42}$. Preclinical data suggest that TNF inhibition mitigates viral pneumonia ${ }^{43}$, and clinical data indicate that patients being treated with TNF inhibitors for inflammatory diseases do not have a worse outcome of COVID-19 than those treated with conventional drugs ${ }^{44}$. Currently, a study evaluating the TNF inhibitor adalimumab in COVID-19 has been registered in the Chinese Clinical Trial Registry (ChiCTR2000030089) ${ }^{45}$.

Beyond the drugs capable of selectively blocking cytokines, much attention has been paid to drugs that modulate the production of more than one cytokine, such as Janus kinase (JAK) inhibitors. As mentioned above, SARS-CoV-2 enters target cells through receptor-mediated endocytosis ${ }^{13}$. Some of the identified regulators of clathrin-mediated endocytosis are members of the numb-associated kinase family, such as AP2-associated protein kinase 1 (AAK1) and cyclin G-associated kinase (GAK). Inhibition of AAK1 might prevent the virus from entering lung cells ${ }^{46}$, and the JAK inhibitor baricitinib is able to effectively inhibit AAK1 and $\mathrm{GAK}^{47}$. Moreover, as an inhibitor of JAK1 and JAK2, baricitinib inhibits IL-6 and GM-CSF, concentrations of which are elevated in COVID-19 (REF. ${ }^{22}$ ). At least five studies with baricitinib, one with tofacitinib and one with ruxolitinib are ongoing ${ }^{48}$. a Viral elimination

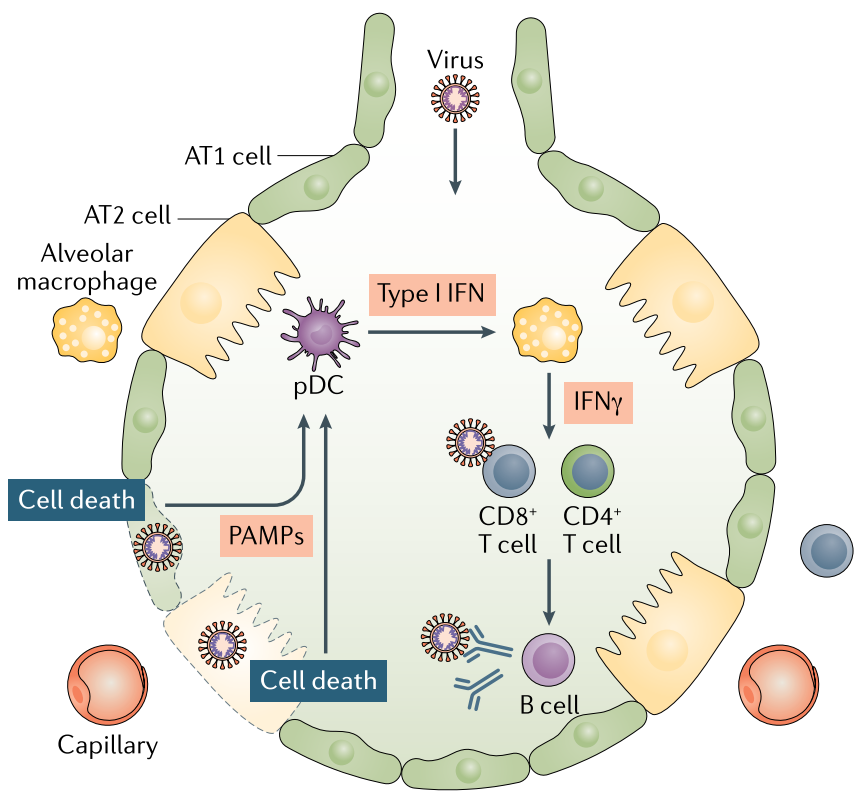

Adaptive immune response leads to viral clearance (resembling mild COVID-19) b Uncontrolled inflammation

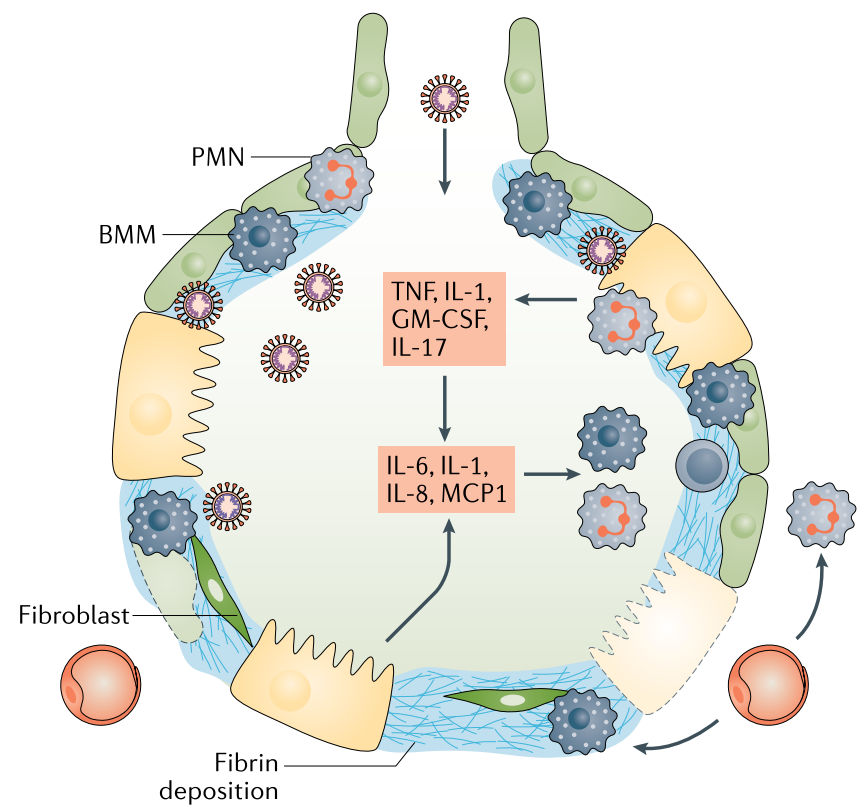

Enhanced innate immune response leads to ARDS (resembling severe COVID-19)

Fig. 2 Alveolar inflammation in COVID-19. a | In mild disease, the adaptive immune response leads to the clearance of severe acute respiratory syndrome coronavirus 2 (SARS-CoV-2) from the lung. b | In severe coronavirus disease 2019 (COVID-19), an excessive or uncontrolled innate immune response leads to a cytokine storm and acute respiratory distress syndrome (ARDS). AT1, alveolar type 1; AT2, alveolar type 2; BMM, bone-marrow-derived macrophage; GM-CSF, granulocyte-monocyte colony-stimulating factor; IFN, interferon; PAMPs, pathogen-associated molecular patterns; pDC, plasmacytoid dendritic cell; PMN, polymorphonuclear granulocyte. 
GM-CSF is a key mediator for macrophage and neutrophil attraction from the bone marrow to inflamed tissues and is a validated target for the treatment of RA. GM-CSF thereby represents a link between $\mathrm{T}$ cell activation and innate immune effector cells, as $\mathrm{T}_{\mathrm{H}} 17$ cells are a major source of $\mathrm{GM}^{-\mathrm{CSF}^{49}}$. As GM-CSF levels are high in COVID-19 ( $\left(\right.$ REF. $\left.^{22}\right)$, studies have been initiated of neutralizing antibodies that block GM-CSF in COVID-19 (REF. $\left.{ }^{50}\right)$.

\section{Cytokine inhibition and viruses}

Although most of the cytokines induced in COVID-19, as well as those being targeted in RA and other forms of inflammatory arthritis, are important in mounting an inflammatory response, they might not be essential for controlling virus clearance. In support of this concept, therapeutic neutralization of TNF, IL- 6 and IL- $1 \beta$ yields a moderate increase in bacterial but not viral infection. Notably, although the incidence of influenza and the risk of developing complications from influenza infection is 2.75-fold higher in patients with RA than in individuals without RA, no signal related to cytokine inhibitor treatment has been observed $^{51}$. In addition, patients with RA undergoing treatment with TNF inhibitors achieve a normal immune response to influenza vaccination, further supporting the concept that effector cytokines induced by SARS-CoV-2 and in RA are critical for inflammatory responses but not for viral clearance. As a matter of fact, data from patients with RA and those with inflammatory bowel disease treated with TNF inhibitors seem to be reassuring, with no increase in the risk of COVID-19 (REFS ${ }^{44,52,53}$ ). However, further data on the adverse, or potentially even beneficial, effects of cytokine inhibitors in respiratory infections need to be collected, as preclinical data (for example, on IL-6) have also suggested that cytokines not only have an important role in the inflammatory response against viruses but are also required to mount a proper immune response against the virus ${ }^{54}$.

In contrast to compounds directed against single pro-inflammatory cytokines, the use of JAK inhibitors might raise some concern, in principle, with respect to viral clearance. Type I interferons are among the most powerful molecules for preventing viral replication. These molecules exert their action via JAK-STAT signalling, which activates interferon-stimulated genes to enable elimination of virus-infected cells. Many viruses have developed strategies to counteract the effects of type I interferons, some of which antagonize the JAK-STAT pathway ${ }^{55}$. Consequently, JAK blockade most likely impairs interferon-mediated

Table 1 | Comparison of mechanistic disease features in COVID-19 and RA

\begin{tabular}{|c|c|c|}
\hline Feature & COVID-19 & RA \\
\hline \multicolumn{3}{|l|}{ Disease features } \\
\hline Triggering factor & Infectious & Autoimmune \\
\hline Target tissue & Alveolar membrane & Synovial membrane \\
\hline Smoking as a risk factor & + & + \\
\hline Disease onset & Acute & Subacute \\
\hline Disease course & Self-limited or lethal & Chronic \\
\hline Effect of glucocorticoids & - & ++ \\
\hline \multicolumn{3}{|l|}{ Contributing cytokines } \\
\hline IL-6 induction & ++ & +++ \\
\hline TNF induction & ++ & +++ \\
\hline GM-CSF induction & ++ & +++ \\
\hline IL-1 induction & ++ & + \\
\hline IL-17 induction & + & $+/-$ \\
\hline \multicolumn{3}{|l|}{ Cellular processes } \\
\hline Resident macrophage defence & ++ & ++ \\
\hline PMN and macrophage infiltration & +++ & ++ \\
\hline T cell infiltration & ++ & ++ \\
\hline Fibrotic response & ++ & ++ \\
\hline \multicolumn{3}{|c|}{$\begin{array}{l}\text { COVID-19, coronavirus disease } 2019 \text {; GM-CSF, granulocyte-monocyte colony-stimulating factor; } \\
\text { PMN, polymorphonuclear granulocyte; RA, rheumatoid arthritis. +/-, little or no effect; +, weak positive } \\
\text { effect; ++, strong positive effect or presence; +++, very strong positive effect or presence; }- \text {, no effect or } \\
\text { negative effect. }\end{array}$} \\
\hline
\end{tabular}

anti-viral responses, which could promote rather than inhibit SARS-CoV-2 infection. This mechanism explains the increased risk of varicella zoster and herpes simplex virus infection reported in patients treated with JAK inhibitors ${ }^{56}$. Notably, viral infections (including herpes zoster and herpes simplex) can account for up to $10 \%$ of community-acquired and up to $50 \%$ of ventilator-associated pneumonia cases ${ }^{57}$. Moreover, lymphocytopenia seems to be a negative prognostic factor in severely affected patients with COVID-19 (REF. ${ }^{58}$ ), and JAK-inhibitor-induced leukopenia is a well-known adverse event. Therefore, while awaiting results from ongoing studies, a word of caution is warranted before suggesting JAK inhibition in this setting ${ }^{59,60}$.

Beyond studies aimed at identifying an effective treatment for COVID-19, the availability of a specific vaccine for SARS-CoV-2 will certainly offer a possible definitive exit from the pandemic. As of 8 April 2020, the global COVID-19 vaccine research and development landscape included 115 vaccine candidates, of which 78 are being actively pursued at the moment ${ }^{61}$. In general, it should be emphasized that, despite many discussions about the safety and efficacy of vaccination in patients receiving immunosuppressive therapy, data from large cohorts indicate that vaccines might be safer and more effective in this setting than previously thought ${ }^{62}$.

\section{COVID-19 effects on rheumatology}

The COVID-19 pandemic is having a profound effect on rheumatology practice. On the one hand, a large part of the usual activity of rheumatology units has been absorbed by the growing need to manage patients with COVID-19; on the other hand, the need to maintain the high standard of care required for the treatment of rheumatic diseases remains ${ }^{63}$. The reduction in the number of physicians available in rheumatology, owing to absence because of disease, quarantine or involvement in the COVID units, is difficult to reconcile with the treat-to-target approach that has now become the core of the treatment of all chronic inflammatory diseases. In addition, ensuring the proper evaluation of patients receiving immunomodulatory drugs and the timely management of disease flares becomes increasingly challenging in this context. Rheumatologists should reorganize their work by trying to postpone all non-urgent visits and weighing the potential harm of delaying an in-person visit against the potential harm of COVID-19 infection. We are learning how to make a 
virtue of necessity by unlocking a new era in patient management. Indeed, the COVID-19 pandemic has suddenly disrupted the traditional model of health care, both for patients with rheumatic diseases and for rheumatologists, accelerating the transition to remote health care ${ }^{64}$.

Finally, the widespread use of antirheumatic drugs in patients with COVID-19 in areas affected by the pandemic, in a fast and sometimes irrationally growing market, could endanger supplies of these drugs for patients with rheumatic diseases ${ }^{65}$. This challenge is already being faced in Europe and the USA regarding the use of hydroxychloroquine, chloroquine and tocilizumab ${ }^{66}$. Regulators and manufacturers will have to keep this problem in mind to avoid unexpected and regrettable disease recurrence in patients with rheumatic disease owing to suspension of treatment.

\section{Conclusions}

The pattern of pro-inflammatory effector cytokines in the alveolar membranes during severe COVID-19 shares similarities with the primary cytokines targeted in the treatment of RA (TABLE 1). Both diseases lead to profound inflammation of structures that form the inner surfaces of the body and induce tissue destruction and responses that lead to organ failure. COVID-19 is characterized by an excessive or uncontrolled host immune response, elicited by alveolar epithelial cell damage and $T$ cell activation in the lungs that triggers exaggerated local production of pro-inflammatory effector cytokines and leads to the attraction of large numbers of neutrophils and macrophages to the lungs. Barrier damage, T cell activation, effector cytokine production and neutrophil influx are also key features of synovitis, and some of the mediators are shared between COVID-19 and RA. The bulk of inflammation and structural damage in COVID-19 is limited to the lungs, which are progressively destroyed. Furthermore, and most likely owing to robust

IL-6 activation, COVID-19 leads to systemic acute-phase responses, which are shared with RA. Whether cytokine inhibition can block this process remains to be determined, but a rationale exists for this approach. Surprisingly, the original widespread concerns about using cytokine inhibitors in a potentially life-threating infectious disease such as COVID-19 have been balanced by the recognition that a pathological hyper-inflammatory state is driving the pathology and might require therapeutic cytokine inhibition. These concepts will also affect our advice to patients with arthritis about whether to stop or continue anti-rheumatic therapy. Given that most of these treatments do not increase the risk of viral pneumonia, that glucocorticoids, which are often used to treat flares, are potentially deleterious in COVID-19, and that preliminary data from high-risk areas do not suggest an increased risk of COVID-19 in patients with arthritis, anti-rheumatic therapy should be continued during the pandemic and not be pre-emptively stopped.

Georg Schett $\mathbb{D}^{1,2 \otimes}$, Bernhard Manger ${ }^{1,2}$, David Simon (ID) 1,2 and Roberto Caporali ${ }^{3}$

'Department of Medicine 3, Friedrich-Alexander University (FAU) Erlangen-Nuremberg and Universitätsklinikum Erlangen, Erlangen, Germany. ${ }^{2}$ Deutsches Zentrum fuer Immuntherapie (DZI), FAU Erlangen-Nuremberg and Universitätsklinikum Erlangen, Erlangen, Germany.

${ }^{3}$ Department of Clinical Sciences and Community Health, Research Center for Adult and Pediatric Rheumatic Diseases, University of Milan. G. Pini Hospital, Milan, Italy.

凶e-mail: Georg.Schett@uk-erlangen.de https://doi.org/10.1038/s41584-020-0451-z

\section{Published online 19 June 2020}

1. Wu, F. et al. A new coronavirus associated with human respiratory disease in China. Nature 579, 265-269 (2020).

2. Wu, Z. \& McGoogan, J. M. Characteristics of and important lessons from the coronavirus disease 2019 (COVID-19) in China. JAMA 323, 1239-1242 (2020).

3. Wichmann, D. et al. Autopsy findings and venous thromboembolism in patients with COVID-19. Ann. Intern. Med. https://doi.org/10.7326/M20-2003 (2020).

4. Hon, K. L. et al. Clinical presentations and outcome of severe acute respiratory syndrome in children. Lancet 361, 1701-1703 (2003)

5. Viner, R. M. \& Whittaker, E. Kawasaki-like disease emerging complication during the COVID-19 pandemic. Lancet 395, 1741-1743 (2020).

6. Marks, M. \& Marks, J. L. Viral arthritis. Clin. Med. 16, 129-134 (2016).

7. Friedman, N. et al. Human coronavirus infections in Israel: epidemiology, clinical symptoms and summer seasonality of HCoV-HKU1. Viruses 10, 515 (2018).

8. Guan, W.-J. et al. Clinical characteristics of coronavirus disease 2019 in China. N. Engl. J. Med. 382, 1708-1720 (2020)

9. Christian, M. D., Poutanen, S. M., Loutfy, M. R. Muller, M. P. \& Low, D. E. Severe acute respiratory syndrome. Clin. Infect. Dis. 38, 1420-1427 (2004).

10. Li, S. et al. Symptom combinations associated with outcome and therapeutic effects in a cohort of cases with SARS. Am. J. Chin. Med. 34, 937-947 (2006).

11. Memish, Z. A., Perlman, S., Van Kerkhove, M. D. \& Zumla, A. Middle East respiratory syndrome. Lancet 395, 1063-1077 (2020)

12. Russel, C. D. et al. Clinical evidence does not support corticosteroid treatment for 2019-nCoV lung injury. Lancet 395, 473-475 (2020).

13. Peiris, J. S. M. et al. Clinical progression and viral load in a community outbreak of coronavirus-associated SARS pneumonia: a prospective study. Lancet 361 1767-1772 (2003)

14. Stockman, L. J., Bellamy, R. \& Garner, P. SARS systematic review of treatment effects. PLoS Med. 3 , e343 (2006)

15. Joo, Y. B., Lim, Y.-H., Kim, K.-J., Park, K.-S. \& Park, Y.-J. Respiratory viral infections and the risk of rheumatoid arthritis. Arthritis Res. Ther. 21, 199 (2019).

16. Hoffmann, M. et al. SARS-CoV-2 cell entry depends on ACE2 and TMPRSS2 and is blocked by a clinically proven protease inhibitor. Cell 181, 271-280.e8 (2020).
17. Pedersen, K. B. et al. Dynamics of ADAM17-mediated shedding of ACE2 applied to pancreatic islets of male $\mathrm{db} / \mathrm{db}$ mice. Endocrinology 156, 4411-4425 (2015)

18. Liu, J. et al. Overlapping and discrete aspects of the pathology and pathogenesis of the emerging human pathogenic coronaviruses SARS-CoV, MERS-CoV, and 2019-nCoV. J. Med. Virol. 92, 491-494 (2020).

19. Katsura, H. et al. IL-1 and TNFa contribute to the inflammatory niche to enhance alveolar regeneration. Stem Cell Rep. 12, 657-666 (2019).

20. Starr, M. et al. Age-associated increase in cytokine production during systemic inflammation - II: the role of IL-1 $\beta$ in age-dependent IL- 6 upregulation in adipose tissue. J. Gerontol. 70, 1508-1515 (2015).

21. Angelidis, I. et al. An atlas of the aging lung mapped by single cell transcriptomics and deep tissue proteomics. Nat. Commun. 10, 963 (2019).

22. Huang, C. et al. Clinical features of patients infected with 2019 novel coronavirus in Wuhan, China. Lancet 395 , 497-506 (2020).

23. Schett, G., Sticherling, M. \& Neurath, M. F. COVID-19 risk for cytokine targeting in chronic inflammatory diseases? Nat. Rev. Immunol. 20, 271-272 (2020).

24. Yoshikawa, T. et al. Severe acute respiratory syndrome (SARS) coronavirus-induced lung epithelial cytokines exacerbate SARS pathogenesis by modulating intrinsic functions of monocyte-derived macrophages and dendritic cells. J. Virol. 83, 3039-3048 (2009).

25. Zhang, X. et al. Nucleocapsid protein of SARS-CoV activates interleukin- 6 expression through cellular transcription factor NF-кB. Virology 365, 324-335 (2007).

26. Law, H. K. et al. Chemokine up-regulation in SARS-coronavirus-infected, monocyte-derived human dendritic cells. Blood 106, 2366-2374 (2005).

27. Josset, L. et al. Cell host response to infection with novel human coronavirus EMC predicts potential antivirals and important differences with SARS coronavirus. $\mathrm{mBio} 4$ e00165-13 (2013)

28. Ryzhakov, G. et al. IL-17 boosts proinflammatory outcome of antiviral response in human cells. J. Immunol. 187, 5357-5362 (2011).

29. Rauber, S. et al. Resolution of inflammation by interleukin-9-producing type 2 innate lymphoid cells. Nat. Med. 23, 938-944 (2017).

30. Chen, Z. et al. $\mathrm{T}_{\mathrm{H}} 2$ and eosinophil responses suppress inflammatory arthritis. Nat. Commun. 7, 11596 (2016).

31. Sarzi-Puttini, P. et al. COVID-19, cytokines and immunosuppression: what can we learn from severe acute respiratory syndrome? Clin. Exp. Rheumatol. 38 , 337-342 (2020).

32. Henderson, L. A. et al. On the alert for cytokine storm immunopathology in COVID-19. Arthritis Rheumatol. https://doi.org/10.1002/art.41285 (2020).

33. Stebbing, J. et al. COVID-19: combining antiviral and anti-inflammatory treatments. Lancet Infect. Dis. 20 , 400-402 (2020).

34. Channappanavar, R. \& Perlman, S. Pathogenic human coronavirus infections: causes and consequences of cytokine storm and immunopathology. Semin Immunopathol. 39, 529-539 (2017).

35. Pedersen, S. F. \& Ho, Y. C. SARS-CoV-2: a storm is raging. J. Clin. Invest. 130, 2202-2205 (2020).

36. Xu, X. et al. Effective treatment of severe COVID-19 patients with tocilizumab. Proc. Natl Acad. Sci. USA 117 10970-10975 (2020).

37. US National Library of Medicine. ClinicalTrials.gov https://clinicaltrials.gov/ct2/show/NCT04380961 (2020).

38. US National Library of Medicine. ClinicalTrials.gov https://www.clinicaltrials.gov/ct2/show/NCT04324021 (2020).

39. US National Library of Medicine. ClinicalTrials.gov https://www.clinicaltrials.gov/ct2/show/NCT04348448 (2020).

40. Qian, Y. et al. Innate immune response of human alveolar type Il cells infected with severe acute respiratory syndrome-coronavirus. Am. J. Respir. Cell Mol. Biol. 48 742-748 (2013)

41. Tobinick, E. TNF- $\alpha$ inhibition for potential therapeutic modulation of SARS coronavirus infection. Curr. Med. Res Opin 20, 39-40 (2004).

42. Feldmann, M. et al. Trials of anti-tumour necrosis factor therapy for COVID-19 are urgently needed. Lancet 395 1407-1409 (2020)

43. Hussell, T., Pennycook, A. \& Openshaw, P. J. Inhibition of tumor necrosis factor reduces the severity of virus-specific lung immunopathology. Eur. J. Immunol. 31, 2566-2573 (2001)

44. Monti, S. et al. Clinical course of COVID-19 in a series of patients with chronic arthritis treated 
with immunosuppressive targeted therapies. Ann. Rheum. Dis. 79, 667-668 (2020).

45. Chinese Clinical Trial Registry. ChiCTR.org http://www. chictr.org.cn/showprojen. aspx? proj=49889 (2020).

46. Lu, R. et al. Genomic characterisation and epidemiology of 2019 novel coronavirus: implications for virus origins and receptor binding. Lancet 395 , 565-574 (2020)

47. Richardson, P. et al. Baricitinib as potential treatment for 2019-nCoV acute respiratory disease. Lancet 395 e30-e31 (2020)

48. US Department of Health and Human Services. Coronavirus (COVID-19). NIH.gov https://www.nih.gov/ health-information/coronavirus (2020).

49. Codarri, L. et al. RORyt drives production of the cytokine GM-CSF in helper T cells, which is essential for the effector phase of autoimmune neuroinflammation. Nat. Immunol. 12, 560-567 (2011).

50. US National Library of Medicine. ClinicalTrials.gov https://clinicaltrials.gov/ct2/show/NCT04341116 (2020).

51. Blumentals, W. A. et al. Rheumatoid arthritis and the incidence of influenza and influenza-related complications: a retrospective cohort study. BMC Musculoskelet. Disord. 13, 158 (2012).

52. An, P. et al. Protection of 318 inflammatory bowel disease patients from the outbreak and rapid spread of COVID-19 infection in Wuhan, China. https:// ssrn.com/abstract=3543590 (Social Science Research Network, 2020).

53. Norsa, L. et al. Uneventful course in IBD patients during SARS-CoV-2 outbreak in northern Italy. Gastroenterology https://doi.org/10.1053/ j.gastro.2020.03.062 (2020).

54. Velazquez-Salinas, L. et al. The role of interleukin- 6 during viral infection. Front. Microbiol. 10, 1057 (2019).
55. Fleming, S. Viral inhibition of the IFN-induced JAK/STAT signalling pathway: development of live attenuated vaccines by mutation of viral-encoded IFN-antagonists. Vaccines 4, 23 (2016).

56. Bechman, K. et al. A systematic review and metaanalysis of infection risk with small molecule JAK inhibitors in rheumatoid arthritis. Rheumatology $\mathbf{5 8}$, 1755-1766 (2019).

57. Kelesidis, T., Mastoris, I., Metsini, A. \& Tsiodras, S. How to approach and treat viral infections in ICU patients. BMC Infect. Dis. 14, 321 (2014).

58. Tan, L. et al. Lymphopenia predicts disease severity of COVID-19: a descriptive and predictive study. Signal. Transduct. Targ. Ther. 5, 33 (2020).

59. Favalli, E. G., Biggioggero, M., Maioli, G. \& Caporali, R. Baricitinib for COVID-19: a suitable treatment? Lancet Infect. Dis. https://doi.org/10.1016/S1473. 3099(20)30262-0 (2020).

60. Praveen, D., Chowdary, P. R. \& Aanandhi, M. V. Baricitinib, a Janus kinase inhibitor, not an ideal option for management of COVID 19. Int. J. Antimicrob. Agents 55, 105967 (2020)

61. Thanh, Le. T. et al. The COVID-19 vaccine development landscape. Nat. Rev. Drug Discov. 19, 305-306 (2020).

62. Meroni, P. L., Zavaglia, D. \& Girmenia, C. Vaccinations in adults with rheumatoid arthritis in an era of new disease-modifying anti-rheumatic drugs. Clin. Exp. Rheumatol. 36, 317-328 (2018)

63. Marotto, D. \& Sarzi-Puttini, P. What is the role of rheumatologists in the era of COVID-19? Autoimmun Rev. 19, 102539 (2020).

64. Hollander, J. E. \& Sites, F. D. The transition from reimagining to recreating health care is now. NEJM Catalyst https://doi.org/10.1056/CAT.20.0093 (2020).
65. Spinelli, F. R., Ceccarelli, F., Di Franco, M. \& Conti, F. To consider or not antimalarials as a prophylactic intervention in the SARS-CoV-2 (Covid-19) pandemic. Ann. Rheum. Dis. 79, 666-667 (2020).

66. European Medicines Agency. COVID-19: chloroquine and hydroxychloroquine only to be used in clinical trials or emergency use programmes. EMA.europa.EU https://www.ema.europa.eu/en/news/covid-19chloroquine-hydroxychloroquine-only-be-used-clinicaltrials-emergency-use-programmes (2020).

\section{Acknowledgements}

The work of G.S. is supported by the German Research Council (DFG: FOR2438/2886; SFB1181; TRR241), the German Ministry of Science and Education (project MASCARA), the European Union (ERC Synergy grant 4DnanoSCOPE) and EU/EFPIA Innovative Medicines Initiative 2 (project RTCure)

\section{Author contributions}

The authors contributed equally to all aspects of the article.

Competing interests

The authors declare no competing interests.

Peer review information

Nature Reviews Rheumatology thanks P. Machado and the other, anonymous, reviewer(s) for their contribution to the peer review of this work.

\section{Publisher's note}

Springer Nature remains neutral with regard to jurisdictional claims in published maps and institutional affiliations.

(c) Springer Nature Limited 2020 